\title{
DE LA STATISTIQUE
}

DU QUÉBEC

\section{Même profession, salaires différents: les femmes professionnelles moins bien rémunérées}

par Maude Boulet ${ }^{1}$

\begin{abstract}
I est bien connu que les femmes sont moins bien rémunérées que les hommes sur le marché du travail (Institut de la statistique du Québec, 2013; Statistique Canada, 2011; Drolet, 2011; Cool, 2010). Par exemple, en 2012, la rémunération horaire moyenne des femmes employées à temps plein était évaluée à 22,35 \$ comparativement à 24,56 \$ chez les hommes du même groupe (figure 1$)^{2}$. Cette figure illustre bien que les femmes n'atteignent toujours pas la parité salariale avec les hommes, mais que ces dernières présentent un ratio de ce salaire grandissant au fil du temps. Ainsi, bien que les femmes gagnent en moyenne un salaire horaire d'environ $10 \%$ inférieur à celui des hommes en 2012, leur rémunération s'est améliorée par rapport à celle des hommes au cours des quinze dernières années au Québec. Autre fait intéressant, les employées québécoises à temps plein sont moins désavantagées par rapport aux hommes que celles de l'ensemble du Canada, et ce, pour toute la période de 1997 à 2012.
\end{abstract}

Récemment, Hango (2013) a établi que, malgré des résultats scolaires comparables en mathématiques au secondaire, les jeunes femmes canadiennes sont moins susceptibles que les jeunes hommes de choisir un programme d'études en sciences, technologies, génie, mathématiques et

sciences informatiques à l'université: des diplômes menant à de bonnes conditions de travail et à une meilleure rémunération sur le marché du travail. Cela peut laisser croire que les femmes choisissent des professions moins bien rémunérées de leur plein gré, ce qui explique leur plus faible rémunération.

Figure 1

Évolution du ratio du salaire horaire moyen des femmes et des hommes chez les employés à temps plein, Québec et Canada, 1997-2012

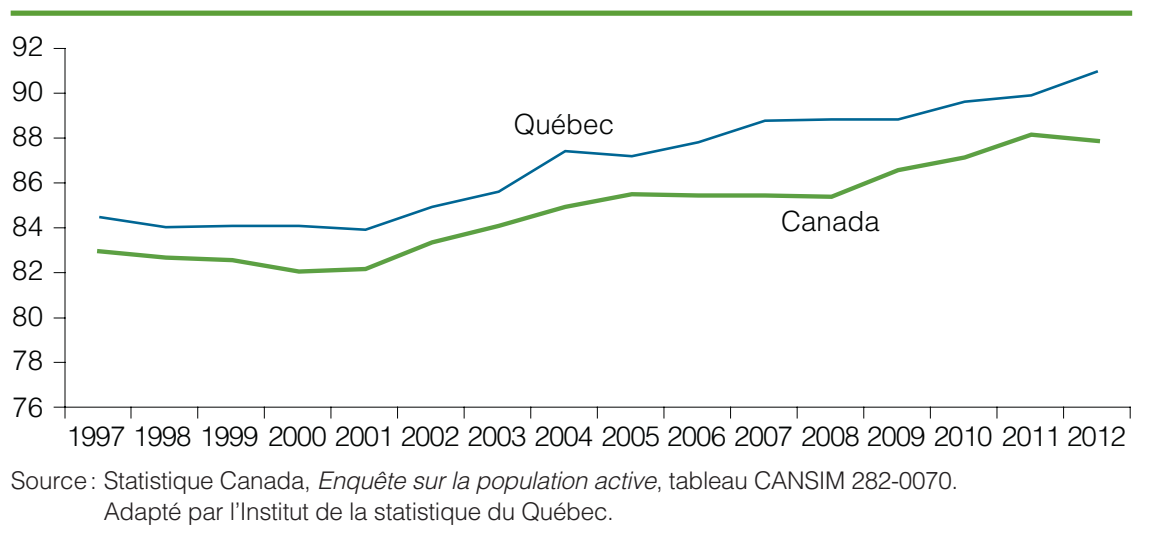

À cet égard, Cool (2010) suggère que les observateurs sont divisés au sujet des raisons de l'écart salarial entre les hommes et les femmes. Certains pensent qu'il résulte non pas d'une discrimination, mais plutôt des choix de carrière des femmes et de leurs décisions en matière de conciliation travailfamille. D'autres soutiennent que les emplois typiquement féminins sont moins bien rémunérés que ceux typiquement masculins parce que les compétences et le travail des femmes ont toujours été sous-estimés. D'ailleurs, Anker et autres (2003) proposent que la séparation des hommes et des femmes dans différentes professions, la ségrégation professionnelle, est l'une des formes les plus pernicieuses d'inégalité sur le marché du travail puisqu'elle s'accompagne de moins bonnes conditions de travail et de rémunération inférieure dans les professions féminines.

Cela met de l'avant la pertinence de comparer la rémunération horaire des hommes et des femmes lorsqu'ils font des choix professionnels similaires. Le tableau 1, tiré des données de l'Enquête sur la population active (EPA), révèle que, chez les employés à temps plein du Québec, les femmes obtiennent un salaire horaire moyen inférieur à celui des hommes dans tous les groupes professionnels, à l'exception du transport et des conducteurs d'équipement lourd. Dans quatre groupes professionnels, la rémunération horaire des femmes est de plus de $25 \%$ inférieure à celle des hommes, notamment les

1. L'auteure remercie Mario Haché de la Direction de la méthodologie et de la qualité pour son soutien méthodologique.

2. En 2013 , le salaire horaire moyen des femmes est de $22,52 \$$ et celui-ci se chiffre à 25,09 \$ chez les hommes. 
cadres supérieurs. L'année dernière, Boulet (2013) a trouvé que des 13300 postes de cadres supérieurs au Québec, plus de 10000 sont occupés par des hommes en 2012. En plus de cet accès limité des femmes aux emplois de cadres supérieurs, les données de l'EPA de 2012 montrent que celles qui réussissent à se hisser aux sommets organisationnels le font avec un salaire horaire moyen plus faible.
Ainsi, les résultats présentés jusqu'ici portent sur l'ensemble des employés à temps plein du Québec. Puisque la profession et la taille de l'entreprise sont reconnues comme des facteurs explicatifs des écarts salariaux entre les hommes et les femmes (Cool, 2010), les données de l'Enquête sur la rémunération globale (ERG) sont également mises à profit afin d'observer la situation des employés réguliers dans les entreprises de 200 employés et plus du Québec. Selon ces données, les hommes gagnent un salaire horaire moyen de 29,16\$ comparativement à $27,77 \$$ chez les femmes pour un écart significatif de 1,39\$ de l'heure.

Tableau 1

Salaire horaire moyen des employés à temps plein selon le groupe professionnel selon la Classification nationale des professions pour statistique (CNP-S) et le sexe, Québec, 2012

\begin{tabular}{|c|c|c|c|}
\hline \multirow[t]{3}{*}{ Groupe professionnel } & \multicolumn{2}{|c|}{ Salaire horaire moyen } & \multirow{3}{*}{$\begin{array}{r}\text { Ratio } \\
\%\end{array}$} \\
\hline & Hommes & Femmes & \\
\hline & \multicolumn{2}{|c|}{$\$$} & \\
\hline Total des employés à temps plein & 24,56 & 22,35 & 91,0 \\
\hline Gestion & 37,55 & 32,30 & 86,0 \\
\hline Cadres supérieurs & 51,81 & 33,91 & 65,5 \\
\hline Autres cadres & 36,46 & 32,24 & 88,4 \\
\hline Affaires, finance et administration & 23,47 & 21,14 & 90,1 \\
\hline Personnel professionnel en gestion des affaires et en finance & 32,19 & 28,72 & 89,2 \\
\hline Personnel en finance, en secrétariat et en administration & 24,53 & 20,72 & 84,5 \\
\hline Personnel de bureau et personnel de supervision du travail de bureau & 19,90 & 19,52 & 98,1 \\
\hline Sciences naturelles et appliquées et professions apparentées & 30,90 & 26,82 & 86,8 \\
\hline Secteur de la santé & 26,67 & 25,01 & 93,8 \\
\hline Personnel professionnel des soins de santé et professionnels en sciences infirmières & 32,82 & 30,83 & 93,9 \\
\hline Personnel technique et de soutien et personnel assimilé du secteur de la santé & 21,48 & 20,54 & 95,6 \\
\hline Sciences sociales, enseignement, administration publique et religion & 31,26 & 27,63 & 88,4 \\
\hline Sciences sociales, administration publique et religion & 28,63 & 25,06 & 87,5 \\
\hline Enseignants & 33,24 & 31,20 & 93,9 \\
\hline Arts, culture, sports et loisirs & 24,04 & 23,34 & 97,1 \\
\hline Ventes et services & 18,83 & 15,18 & 80,6 \\
\hline $\begin{array}{l}\text { Personnel de la vente en gros technique et non technique, de l'assurance et de l'immobilier, } \\
\text { des achats en gros et au détail et des achats de grains }\end{array}$ & 23,94 & 20,78 & 86,8 \\
\hline Personnel de supervision, vendeurs, commis-vendeurs et caissiers des produits au détail & 17,62 & 13,53 & 76,8 \\
\hline Chefs et cuisiniers, personnel de supervision et autre personnel des services des aliments et boissons & 14,97 & 14,03 & 93,7 \\
\hline Personnel des services de protection & 24,65 & 21,85 & 88,6 \\
\hline Personnel de soutien familial et de garderie & 16,83 & 15,35 & 91,2 \\
\hline $\begin{array}{l}\text { Personnel de supervision des services, personnel de l'hébergement et des voyages, } \\
\text { préposés dans les sports et les loisirs et personnel de la vente et des services }\end{array}$ & 16,31 & 14,11 & 86,5 \\
\hline Métiers, transport et machinerie & 22,59 & 19,12 & 84,6 \\
\hline Entrepreneurs et contremaîtres du personnel des métiers et des transports & 27,56 & 24,42 & 88,6 \\
\hline Personnel des métiers de la construction & 25,32 & 20,79 & 82,1 \\
\hline Autres métiers & 23,69 & 17,75 & 74,9 \\
\hline Transport et conducteurs d'équipement lourd & 20,36 & 20,70 & 101,7 \\
\hline Aides de soutien des métiers, manœuvres en construction et de transport et personnel assimilé & 18,83 & 14,91 & 79,2 \\
\hline Professions propres au secteur primaire & 18,28 & 12,62 & 69,0 \\
\hline Transformation, fabrication et services d'utilité publique & 19,80 & 15,20 & 76,8 \\
\hline Surveillants, conducteurs de machines et monteurs dans la fabrication & 20,15 & 15,99 & 79,4 \\
\hline Manoeuvres dans la fabrication et les services d'utilité publique & 17,87 & 13,28 & 74,3 \\
\hline
\end{tabular}

Source: Statistique Canada, Enquête sur la population active, tableau CANSIM 282-0070. Adapté par l'Institut de la statistique du Québec. 


\section{Note méthodologique - deux populations à l'étude distinctes}

Les résultats présentés à la figure 1 et au tableau 1 sont tirés du tableau CANSIM 282-0070 portant sur les données de l'Enquête sur la population active (EPA) de Statistique Canada. Pour ces résultats, la population à l'étude constitue l'ensemble des employés à temps plein au Québec et au Canada. Cette enquête « individu » offre l'avantage de dresser un portrait global de la rémunération horaire des employés selon le sexe sur le marché du travail québécois. Pour ces données tirées du site Internet de Statistique Canada, les coefficients de variation (CV) et les tests de différences statistiques n'ont pas pu être produits.

Pour leur part, les tableaux 2 à 4 sont tirés de l'Enquête sur la rémunération globale (ERG) menée par l'Institut auprès des entreprises de 200 employés et plus. Aux fins de la présente capsule, seuls les employés réguliers à temps plein de ces entreprises font partie de la population étudiée. Cette enquête «entreprise ", qui porte sur la rémunération en vigueur le $1^{\text {er }}$ avril 2012, vient compléter le portrait en mettant l'accent sur l'écart salarial entre les hommes et les femmes du même groupe professionnel dans les entreprises de plus grandes tailles qui possèdent généralement des structures salariales bien établies. Pour les estimations réalisées à partir de l'ERG, la mesure de précision utilisée est le coefficient de variation (CV). Les estimations dont le CV est inférieur ou égal à $15 \%$ sont considérées comme suffisamment précises pour être présentées sans commentaire. De plus, le seuil de $5 \%$ a été utilisé pour identifier les écarts salariaux significatifs entre les hommes et les femmes.

La combinaison des données provenant de deux sources permet d'analyser la situation salariale des femmes sous des angles différents.

Pour plus de détail sur la méthodologie de ces deux enquêtes, veuillez consulter les rapports « Méthodologie de l'Enquête sur la population active du Canada» ainsi que le rapport «Résultats de l'Enquête sur la rémunération globale au Québec, Collecte 2012».

L'écart salarial entre les hommes et les femmes peut résulter d'une différence dans le niveau requis de compétences par leur emploi. À partir des données de l'ERG, le tableau 2 révèle que, peu importe le niveau de compétences de l'emploi occupé, les femmes employées régulières à temps plein obtiennent un salaire horaire moyen plus faible que celui de leurs homologues masculins. Chez les gestionnaires, les femmes gagnent environ 3,61 \$ de l'heure de moins que les hommes. Ces écarts se chiffrent respectivement à $3,33 \$, 3,63 \$, 2,11 \$$ et $2,05 \$$ pour les niveaux de compétences A - Formation universitaire, B - Formation collégiale ou programme d'apprentissage, $\mathrm{C}$ - Formation de niveau secondaire ou formation spécifique à la profession et D - Formation en cours d'emploi. Le ratio salarial moyen est de $90 \%$, lorsqu'on tient compte du niveau de compétences, ce qui va dans le même sens que ce qui a été observé avec les données de l'EPA.

Un examen plus fin des écarts salariaux entre les hommes et les femmes est mené à bien à partir de la Classification nationale des professions à trois chiffres (CNP3) de 2006, puisque plusieurs pro-
Tableau 2

Salaire horaire moyen selon le niveau de compétences et le sexe des employés réguliers à temps plein dans les entreprises de 200 employés et plus (\$), Classification nationale des professions, Québec, 2012

\begin{tabular}{|c|c|c|c|c|}
\hline \multirow[t]{3}{*}{ Niveau de compétences } & \multicolumn{2}{|c|}{ Salaire horaire moyen } & \multirow{3}{*}{$\begin{array}{l}\text { Écart } \\
\text { femmes- } \\
\text { hommes }\end{array}$} & \multirow{3}{*}{$\begin{array}{r}\text { Ratic } \\
\%\end{array}$} \\
\hline & Hommes & Femmes & & \\
\hline & \multicolumn{2}{|c|}{$\$$} & & \\
\hline Gestion $[0]^{1}$ & 48,49 & 44,88 & $-3,61^{\dagger}$ & 92,6 \\
\hline Formation universitaire [A] & 39,82 & 36,49 & $-3,33^{+}$ & 91,6 \\
\hline $\begin{array}{l}\text { Formation collégiale ou programme } \\
\text { d'apprentissage }[B]\end{array}$ & 29,17 & 25,54 & $-3,63^{\dagger}$ & 87,6 \\
\hline $\begin{array}{l}\text { Formation de niveau secondaire ou } \\
\text { formation spécifique à la profession [C] }\end{array}$ & 21,45 & 19,34 & $-2,11^{\dagger}$ & 90,2 \\
\hline Formation en cours d'emploi [D] & 17,78 & 15,73 & $-2,05^{\dagger}$ & 88,5 \\
\hline
\end{tabular}

† Écart significatif au seuil de $5 \%$.

1. Les cadres supérieurs ne sont pas inclus dans ce groupe.

Note: Toutes les estimations présentées ont un coefficient de variation inférieur à 15\%.

Source: Institut de la statistique du Québec, Enquête sur la rémunération globale au Québec. Collecte 2012.

fessions se retrouvent à l'intérieur d'un même niveau de compétences. Cet examen permet d'avoir une idée plus précise de l'écart salarial entre les hommes et les femmes exerçant la même profession.
Par ailleurs, l'analyse porte sur le niveau de compétences $A$, qui requiert habituellement une formation universitaire, pour mettre en évidence la situation salariale relative des femmes professionnelles. 
Le tableau 3 présente dix professions du niveau de compétences $A$, dont les cinq premières sont typiquement masculines et les cinq suivantes sont typiquement féminines. En effet, selon l'article 55 de la Loi sur l'équité salariale, une catégorie d'emplois peut être considérée à prédominance féminine ou masculine lorsqu'au moins $60 \%$ des salariés qui occupent l'emploi en cause sont du même sexe ${ }^{3}$.

À partir de ce critère, on voit notamment qu'à l'exception des professionnels en informatique, le salaire horaire moyen des professions typiquement masculines est supérieur à celui des professions typiquement féminines. En outre, on observe que les femmes sont plus présentes dans les professions de la santé, de l'enseignement et en relations d'aide et que les hommes sont plus concentrés dans les sciences pures et appliquées, ce qui appuie l'étude de Hango (2013) concernant les choix professionnels des jeunes femmes qui sont moins orientées vers les professions en sciences, technologies, génie, mathématiques et sciences informatiques.

Par rapport à cette dernière étude, la nôtre vient ajouter que, même lorsque les femmes occupent une profession typiquement masculine, elles ont généralement une rémunération horaire inférieure (tableau 4). Le tableau 4 fait état de ce constat pour trois professions typiquement masculines, soit les professeures d'université et assistantes d'enseignement au niveau postsecondaire (-8\$/heure), les professionnels en génie civil, mécanique, électrique et chimique $(-2,72 \$$ / heure) ainsi que chez les autres professionnels en génie (-2,71\$/heure). D'un autre côté, on peut aussi bien se demander ce qui advient des écarts salariaux entre les hommes et les femmes dans les emplois typiquement féminins. Le constat est similaire puisque, même lorsque les hommes exercent une profession typiquement féminine, ils gagnent un salaire horaire supérieur à celui des femmes. C'est le cas notamment chez les enseignants aux niveaux secondaire, primaire et préscolaire et les conseillers d'orientation, les professionnels en psychologie, en travail social, en counseling, en religion et en probation ainsi que les professionnels en thérapie et en évaluation.

Tableau 3

Taux de présence féminine et salaire horaire moyen des employés réguliers à temps plein selon la profession dans les entreprises de 200 employés et plus, Classification nationale des professions, niveau de compétences A - Formation universitaire, Québec, 2012

\begin{tabular}{|c|c|c|c|c|}
\hline $\begin{array}{l}\text { Code } \\
\text { CNP3 }\end{array}$ & Titre d'emploi & $\begin{array}{r}\text { Taux de } \\
\text { présence } \\
\text { féminine }\end{array}$ & 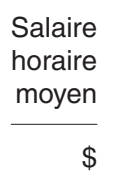 & $\begin{array}{r}\text { Intervalle de } \\
\text { confiance }\end{array}$ \\
\hline 213 & $\begin{array}{l}\text { Professionnels en génie civil, mécanique, } \\
\text { électrique et chimique }\end{array}$ & 17,2 & 40,64 & {$[39,92-41,36]$} \\
\hline 214 & Autres professionnels en génie & 17,5 & 41,81 & {$[41,20-42,41]$} \\
\hline 217 & Professionnels en informatique & 28,1 & 37,76 & {$[36,90-38,63]$} \\
\hline 215 & $\begin{array}{l}\text { Professionnels en architecture, en urbanisme } \\
\text { et en arpentage }\end{array}$ & 35,9 & 41,07 & {$[40,47-41,66]$} \\
\hline 412 & $\begin{array}{l}\text { Professeurs d'université et assistant } \\
\text { d'enseignement au niveau postsecondaire }\end{array}$ & 38,0 & 52,77 & {$[52,51-53,04]$} \\
\hline 414 & $\begin{array}{l}\text { Enseignant aux niveaux secondaire, primaire } \\
\text { et préscolaire et conseiller d'orientation }\end{array}$ & 74,2 & 36,56 & {$[36,46-36,67]$} \\
\hline 511 & $\begin{array}{l}\text { Professionnels des bibliothèques, des archives, } \\
\text { des musées et des galeries d'art }\end{array}$ & 74,4 & 39,29 & {$[39,10-39,49]$} \\
\hline 415 & $\begin{array}{l}\text { Professionnels en psychologie, en travail social, } \\
\text { en counseling, en religion et en probation }\end{array}$ & 79,7 & 36,66 & {$[36,50-36,82]$} \\
\hline 315 & Personnel professionnel en soins infirmiers & 87,9 & 34,21 & {$[34,20-34,23]$} \\
\hline 314 & Professionnels en thérapie et en évaluation & 89,5 & 36,43 & {$[36,23-36,63]$} \\
\hline
\end{tabular}

Note: Toutes les estimations présentées ont un coefficient de variation inférieur à $15 \%$.

Source: Institut de la statistique du Québec, Enquête sur la rémunération globale au Québec. Collecte 2012.

Toutefois, le personnel professionnel en soins infirmiers fait exception à la règle générale, puisque les femmes présentent un avantage salarial de 0,22\$ l'heure.

Ainsi, chez les professionnels dans les entreprises de 200 employés et plus, les femmes ont une rémunération horaire plus faible que celle des hommes. II faut souligner que, si les femmes professionnelles affichent un désavantage salarial par rapport aux hommes dans douze des dixneuf professions présentées au tableau 3 , il peut exister une certaine hétérogénéité à l'intérieur même d'un même code de la CNP3. Par exemple, chez les juges, les avocats et les notaires, l'écart de salaire horaire moyen des juges dépasse 130 , tandis que celui des avocats et des notaires se situe davantage aux alentours de 50\$ (données non présentées). Puisque les femmes sont beaucoup plus présentes parmi les avocats et les notaires que chez les juges (environ $60 \%$ c. $30 \%$ ), une part importante de l'écart salarial entre les hommes et les femmes résulte de ce fait (données non présentées). La situation est semblable chez les professeurs d'université et les assistants d'enseignement au niveau postsecondaire. En revanche, dans certains codes CNP3, par exemple, ceux regroupant les professionnels en génie, les emplois sont plus homogènes.

En somme, cette capsule vient appuyer une imposante littérature montrant que l'écart salarial entre les hommes et les femmes persistent encore aujourd'hui; tant les données de l'ERG que celles de I'EPA conduisent à cette constatation. II est donc légitime de se demander d'où provient cet écart. Selon Cool (2010), les structures salariales reflètent plusieurs facteurs en lien avec le capital humain - expérience de travail, scolarité, durée d'emploi -, les caractéristiques démographiques - situation de la famille, présence d'enfants - et les caractéristiques de l'emploi - couverture syndicale, travail

3. L.R.Q., chapitre E-12.001.

[4] Mars 2014 
Tableau 4

Salaire horaire moyen des employés réguliers à temps plein selon la profession et le sexe dans les entreprises de 200 employés et plus, Classification nationale des professions, niveau de compétences A - Formation universitaire, Québec, 2012

\begin{tabular}{|c|c|c|c|c|c|}
\hline \multirow[t]{3}{*}{$\begin{array}{l}\text { Code } \\
\text { CNP3 }\end{array}$} & \multirow[t]{3}{*}{ Titre d'emploi } & \multicolumn{2}{|c|}{$\begin{array}{c}\text { Salaire horaire } \\
\text { moyen }\end{array}$} & \multirow{3}{*}{$\begin{array}{r}\text { Écart } \\
\text { femmes- } \\
\text { hommes }\end{array}$} & \multirow{3}{*}{$\begin{array}{r}\text { Ratio } \\
\%\end{array}$} \\
\hline & & Hommes & Femmes & & \\
\hline & & \multicolumn{2}{|r|}{$\$$} & & \\
\hline 112 & Professionnels en gestion des ressources humaines et en services aux entreprises & 38,13 & 36,60 & $-1,53^{\dagger}$ & 96,0 \\
\hline 211 & Professionnels des sciences physiques & 40,77 & 36,39 & $-4,38$ & 89,3 \\
\hline 212 & Professionnels des sciences de la vie & 38,23 & 33,06 & $-5,18^{+}$ & 86,5 \\
\hline 213 & Professionnels en génie civil, mécanique, électrique et chimique & 41,11 & 38,39 & $-2,72^{\dagger}$ & 93,4 \\
\hline 214 & Autres professionnels en génie & 42,28 & 39,57 & $-2,71^{\dagger}$ & 93,6 \\
\hline 215 & Professionnels en architecture, en urbanisme et en arpentage & 41,41 & 40,47 & $-0,94$ & 97,7 \\
\hline 216 & Mathématiciens, statisticiens et actuaires & 39,39 & 38,46 & $-0,93$ & 97,6 \\
\hline 217 & Professionnels en informatique & 37,95 & 37,28 & $-0,66$ & 98,2 \\
\hline 314 & Professionnels en thérapie et en évaluation & 37,47 & 36,31 & $-1,16^{+}$ & 96,9 \\
\hline 315 & Personnel professionnel en soins infirmiers & 34,02 & 34,24 & $+0,22^{\dagger}$ & 100,6 \\
\hline 411 & Juges, avocats et notaires & 75,60 & 57,72 & $-17,89^{\dagger}$ & 76,3 \\
\hline 412 & Professeurs d'université et assistant d'enseignement au niveau postsecondaire & 55,88 & 47,71 & $-8,17^{\dagger}$ & 85,4 \\
\hline 413 & Enseignant au niveau collégial et autres instructeurs en formation professionnelle & 37,47 & 37,39 & $-0,08$ & 99,8 \\
\hline 414 & Enseignant aux niveaux secondaire, primaire et préscolaire et conseiller d'orientation & 37,09 & 36,38 & $-0,71^{\dagger}$ & 98,1 \\
\hline 415 & Professionnels en psychologie, en travail social, en counseling, en religion et en probation & 37,47 & 36,45 & $-1,02^{\dagger}$ & 97,3 \\
\hline 416 & Agents des politiques et des programmes, recherchistes, et experts-conseils & 39,52 & 37,25 & $-2,27^{\dagger}$ & 94,3 \\
\hline 511 & Professionnels des bibliothèques, des archives, des musées et des galeries d'art & 39,25 & 39,30 & $+0,05$ & 100,1 \\
\hline 512 & Professionnels de la rédaction, de la traduction et des relations publiques & 37,16 & 34,21 & $-2,95^{\dagger}$ & 92,1 \\
\hline 513 & Professionnels des arts plastiques et des arts de la scène & 38,22 & 37,21 & $-1,01$ & 97,4 \\
\hline
\end{tabular}

† Écart significatif au seuil de $5 \%$.

Notes: Toutes les estimations présentées ont un coefficient de variation inférieur à $15 \%$.

Quatre emplois de niveau de formation universitaire ne font pas partie de l'analyse. Le code CNP 111 est exclus parce qu'une part importante de la rémunération de cette profession est basée sur des commissions. Les codes CNP 311, 312 et 313 sont aussi exclus parce que les résultats relatifs aux salaires ne reflétaient pas la réalité de la profession en raison du mode particulier de rémunération des ses professions, par exemple, la rémunération à l'acte.

Source: Institut de la statistique du Québec, Enquête sur la rémunération globale au Québec. Collecte 2012.

à temps partiel, profession, secteur d'activité et taille de l'entreprise. Dans une étude canadienne, Drolet (2001) a conclu que la moitié de l'écart salarial entre les hommes et les femmes demeurait inexpliqué malgré la prise en compte de tous ces facteurs. Cela signifie notamment que le nombre plus faible d'années d'expérience de travail des femmes et leur concentration dans des professions moins rémunératrices ne sont pas les seuls motifs de leur désavantage salarial.
Bien que la présente étude ne permet pas d'identifier les causes de l'écart de rémunération entre les hommes et les femmes, elle permet tout de même d'établir trois principaux constats. Premièrement, les résultats montrent que le ratio salarial des Québécoises et des Canadiennes (par rapport à leurs homologues masculins respectifs) a augmenté depuis les quinze dernières années; les Québécoises faisant mieux que les Canadiennes. Deuxièmement, les résultats indiquent que les professions typiquement féminines offrent en moyenne une rémunération inférieure à celle des professions typiquement masculines. Enfin, troisièmement, les analyses révèlent que l'écart salarial entre les hommes et les femmes existe généralement au sein d'une même profession. Cet écart salarial porte à réflexion. Même lorsque les femmes s'orientent vers une carrière typiquement masculine comme le génie, leur rémunération est moins élevée. 


\section{Bibliographie}

ANKER, Richard, Helinä MELKAS et Ailsa KORTEN (2003). Gender-based occupational segregation in the 1990s, Genève, International Labour Office, Working paper no 16, $32 \mathrm{p}$.

BOULET, Maude (2013). «L'accès limité des femmes aux emplois de gestion: un plafond de verre? », Institut de la statistique du Québec, 3 p.

COOL, Julie (2010). L'écart salarial entre les femmes et les hommes. Gouvernement du Canada, Bibliothèque du Parlement, Ottawa, publication $n^{\circ}$ 2010-30-F, $11 \mathrm{p}$.

DROLET, Marie (2011). «Pourquoi l'écart salarial entre les hommes et les femmes a-t-il diminué? », L'emploi et le revenu en perspectives, Statistique Canada, $14 \mathrm{p}$.

DROLET, Marie (2001). L'écart persistant: nouvelle évidence empirique concernant l'écart salarial entre les hommes et les femmes au Canada, Direction des études analytiques: document de recherche, Statistique Canada, Ottawa, n 157, 37 p.

HANGO, Darcy (2013). «Les différences entre les sexes dans les programmes de sciences, technologies, génie, mathématiques et sciences informatiques (STGM) à l'université », Regards sur la société canadienne, Statistique Canada, Ottawa, nº 75-006-X au catalogue, 13 p.

INSTITUT DE LA STATISTIQUE DU QUÉBEC (2013). Annuaire québécois des statistiques du travail. Portrait des principaux indicateurs du marché et des conditions de travail, 2002-2012, volume 9, Québec, 264 p.

STATISTIQUE CANADA (2011). Femme au Canada: rapport statistique fondé sur le sexe, sixième édition, Statistique Canada, Ottawa, $n^{\circ}$ 89-503-X au catalogue, $405 \mathrm{p}$.

Ce bulletin est réalisé par la Direction des statistiques du travail et de la rémunération.

Ont collaboré à la réalisation: Esther Frève, révision linguistique

Gabrielle Tardif, mise en page

Direction des communications

Pour plus de renseignements: Maude Boulet, analyste en statistique du travail

Direction des statistiques du travail et de la rémunération

1200, avenue McGill College, $4^{e}$ étage

Montréal (Québec) H3B 4J8

Téléphone: $514876-4384$

Télécopieur : 514 876-1767

Courriel : $\quad$ publication.dstr@stat.gouv.qc.ca

Toute reproduction autre qu'à des fins de consultation personnelle est interdite sans l'autorisation du gouvernement du Québec. www.stat.gouv.qc.ca/droits_auteur.htm 